\title{
The effects of thrombolytic therapy on prognostic clinical markers in patients with intermediate-high risk pulmonary embolism
}

\author{
Alexandru Cristian Ion ${ }^{1,2}$, Stefan Busnatu, ${ }^{1,2}$, Catalina Liliana Andrei ${ }^{1,2}$, \\ Cristinel Dumitru Badiu ${ }^{1,3}$, Crina Julieta Sinescu, ${ }^{1,2}$ \\ 1"Carol Davila" University of Medicine and Pharmacy, Bucharest, Romania \\ ${ }^{2}$ Cardiology Department, "Bagdasar-Arseni" Emergency Hospital, Bucharest, Romania \\ ${ }^{3}$ Surgery Department, "Bagdasar-Arseni“ Emergency Hospital, Bucharest, Romania
}

\begin{abstract}
The objective of the study is to assess the efect of thrombolitic therapy on clinical prognostic markers in patients with intermediate-high risk pulmonary embolism compared to clasic therapy, and also the assessment of the bleeding risk associated with thrombolitic therapy.

Material and methods. We included 82 consecutive patients with intermediate-high risk pulmonary embolism, which were divided in two groups: study group (thrombolysed) and control group (treated with unfractioned heparine alone). Patients with lower bleeding risk were included in control group. Patients were assessed regarding the right ventricle dysfunction markers (both imagistic and biochemical) on admision and on $48 \mathrm{~h}$. The early (7th day) mortality rate and hemodinamic instability rate were evaluated in the two groups, also the major bleeding rate.

Outcomes. We proved a statistical significant lower mortality rate in the study group, with no difference regarding the major bleedings. Meanwhile, the hemodinamic instability was lower in the study group compared to control group.

Conclusions. The proper patient selection with intermediate-high pulmonary embolism for thrombolitic therapy can bring important benefits in terms of mortality and bleeding complications.
\end{abstract}

Keywords: pulmonary emobolism, intermediary-high risk, trombolysis, mortality rate, thrombolitic therapy

\author{
Abbreviations used \\ PE - pulmonary embolism \\ DVT - deep venous thrombosis \\ BNP - brain natriuretic peptide \\ $\mathrm{Nt}$ pro $\mathrm{BNP}-\mathrm{N}$ terminal pro brain natriuretic peptide \\ $\mathrm{RV}$ - right ventricle \\ PESI - pulmonary embolism severity index \\ VTE - venous thromboembolism \\ ESC - European Society of Cardiology \\ TAPSE - tricuspid annulus pick systolic excursion \\ $\mathrm{MCV}$ - myocardial contraction velocity
}

\author{
$\mathrm{LV}$ - left ventricle \\ t-PA - tissue plasminogen activator (alteplase) \\ aPTT - partially activated prothrombin time \\ UFH - unfractionated heparine \\ BMI - body mass index \\ ISTH - International Society of Thrombosis and Hemostasis \\ SBP - systolic blood presure \\ $\mathrm{HR}$ - heart rate \\ COPD - chronic obstructive pulmonary disease \\ $\mathrm{CHF}$ - chronic heart failure
}

\section{BACKGROUND}

Pulmonary embolism (PE) is a medical condition with an increasing incidence, given the increasing frequency of predisposing risk factors.
Together with deep vein thrombosis (DVT) is a clinical continuum, explained by the fact that the most common source of thrombi in the pulmonary arterial system is found in the deep venous system. 
The predisposing factors for DVT and PE act through one or more elements of the Virchow triad: hypercoagulability, venous stasis, endothelial dysfunction. Intermediate-high risk PE is defined by both biochemical (brain natriuretic peptide (BNP) or $\mathrm{N}$ terminal BNP (Nt pro BNP)) and imaging right ventricular dysfunction (RV) markers (RV pressure overload echocardiographic markers), without the haemodynamic instability. Meanwhile, the clinical classification of PE severity by Pulmonary Embolism Severity Index (PESI score) includes in intermediate category patients with PESI score $>90$ (PESI class $>$ III).

In six European countries with a total population of 454.6 million, more than 37,000 deaths were caused by venous thromboembolism (VTE) in 2004, according to estimates from an epidemiological model (1). Of these patients, 34\% died suddenly before therapy was initiated or was effective. From these patients $59 \%$ were correctly diagnosed with postmortem PE (1). In the US, EP has caused about 300,000 deaths/year, being responsible for one of the highest cardiovascular deaths (2). Epidemiological analyzes performed in European, Asian and North American populations suggest that mortality rates in acute PE may be declining $(3,4)$. The increasing use of effective therapies and better adherence to current guidelines may contribute to this outcome $(5,6)$. However, there is a tendency to overdiagnose PE (subsegmental or even non-existent) (7), and this fact can induce a false decrease in mortality rates.

The role of classifying the risk of pulmonary embolism lies in the implications that this stratification has on acute therapy. The Guidelines of the European Society of Cardiology (ESC) for the Management of Pulmonary Embolism (2019) recommends thrombolytic therapy to patients at intermediate risk in case of hemodynamic instability (8). It should be taken into account that the main factor limiting the use of thrombolytic therapy in patients with intermediate-risk $\mathrm{PE}$ is the associated bleeding risk. Hemodynamic instability is defined as a decrease in systolic blood pressure values by more than $40 \mathrm{mmHg}$ compared to the initial assessment or below $90 \mathrm{mmHg}$, associating signs of peripheral hypoperfusion. Regarding thrombolytic therapy in normotensive patients, the PEITHO study showed a statistically significant decrease in the rate of hemodynamic instability in thrombolyzed patients compared to classically treated patients, with an increased rate of hemorrhagic complications (9). A meta-analysis of 7 studies comparing the impact of thrombolytic therapy on mortality and hemodynamic instability in patients with inter- mediate-high risk $\mathrm{PE}$ showed a lower mortality rate in the thrombolyzed group, without statistical significance (OR 0.15, P value 0.23) $(10,11)$. Also, in the PEITHO study, the rate of hemorrhagic complications was 10 times higher in the thrombolyzed group compared to the classic treated group in patients over 80 years, with severe renal dysfunction, obesity or underweight (2). It is therefore obvious the need to further investigate the effects of thrombolytic therapy in patients with intermediate-high risk $\mathrm{PE}$, taking into account the classes of hemorrhagic risk resulting from existing evidence and assessing the effects on the markers of right ventricular dysfunction and clinical parameters - mortality, haemodynamic instability.

\section{MATERIAL AND METHODS}

The main objective of this study is the comparative analysis of mortality and hemodynamic instability in the group of thrombolyzed intermediatehigh risk PE patients compared to classically treated patients. The secondary objective is represented by the safety element, regarding the rate of major bleedings.

In this study we enrolled patients with acute intermediate-high risk PE, defined by PESI clinical severity score $>90$, ultrasound signs of RV pressure overload (revealed either by RV dilation due to RV pressure overload, by longitudinal RV systolic dysfunction (evaluated by tricuspid annular peak systolic excursion (TAPSE) or by decreasing myocardial contraction velocity (MCV)) and RV dysfunction revealed by biological markers $(\mathrm{BNP}>$ $150 \mathrm{pg} / \mathrm{ml}$ ).

The selected patients had to match the inclusion criteria: 1. The first documented episode of acute pulmonary embolism; 2 . Age over 18 years; 3 . The subjects understand the study and agree to be part of it - informed consent.

Exclusion criteria were: 1. Increased risk of bleeding secondary to blood disorders: hemophilia, severe thrombocytopenia $<50.000 / \mathrm{mm}^{3} ; 2$. Severe left ventricular (LV) systolic dysfunction assessed by LV ejection fraction $<30 \%$; 3 . Severe valvulopathies possibly associated with secondary pulmonary hypertension: severe mitral stenosis, significant mitral regurgitation, severe aortic stenosis, severe aortic regurgitation; 4. Previous pulmonary embolism; 5. Diseases with life expectancy less than 1 year: terminal neoplasms; 6. Persons not able to exercise informed consent; 7. Age over 85 years; 8 . Severe anemic syndromes, with seric haemoglobin $<9 \mathrm{~g} / \mathrm{dl}$. 
We enrolled in this study 82 consecutive patients with intermediate-high risk PE defined according to the ESC criteria, meeting the inclusion criteria and without exclusion criteria. Patients over 85 years old were excluded from the study because of an increased bleeding risk, which was proven to be associated with anticoagulant and thrombolytic therapy. Patients were divided into 2 groups, study group - 39 patients (receiving thrombolytic therapy t-PA $100 \mathrm{mg}$ in 2 hours combined with anticoagulant therapy - unfractionated heparin (UFH) dosed depending on the activated partial thromboplastin time (aPTT)) and control group - 43 patients (treated with UFH dosed according to aPTT). The control group included patients with contraindications for thrombolytic therapy and patients in whom, according to current evidence, thrombolytic therapy is associated with an increased risk of bleeding (obese (body mass index $(\mathrm{BMI})>30 \mathrm{~kg} / \mathrm{m}^{2}$ ) and underweight (BMI $<18.5 \mathrm{~kg} / \mathrm{m}^{2}$ ), severe renal dysfunction - creatinine clearance $<30 \mathrm{ml} / \mathrm{min} / \mathrm{m}^{2}$ ). Patients were evaluated on admission, in terms of the clinical severity of PE, by the PESI score, as well regarding the incidence of the main risk factors. The evaluation of RV overload markers included BNP dosing at admission, evaluation of echocardiographic signs of RV overload (RV dilation, supraunitary $\mathrm{RV} / \mathrm{LV}$ ratio, TAPSE, MCV). Subsequently, at $48 \mathrm{~h}$, the echocardiographic reassessment followed the dynamics of the same markers, and the BNP reassessment at $72 \mathrm{~h}$ followed the evolution of BNP. For the analysis of clinical prognostic markers, we followed the rate of early mortality (at 7 days) and early hemodynamic instability (at 7 days). To analyze the bleeding risk of thrombolytic therapy, we monitored the rate of major bleeding (defined according to the International Society of Hemostasis and Thrombosis (ISTH)). According to this definition, the major events include fatal hemorrhages, hemorrhages in some parenchymal organs (brain, gastroenterological, retroperitoneal, intraspinal), as well as hemorrhages causing a decrease in hemoglobin values by more than $2 \mathrm{~g} / \mathrm{dl}$ compared to baseline. Statistical analysis was performed using SPSS statistical program, and the use of Chi-square statistical significance tests, Welch T test, Wilcoxon test and Mann-Whitney test depending on the normality of data distribution - evaluated by the Shapiro-Wilk test. The research was conducted in compliance with the ethical principles of the national committee.

\section{RESULTS}

The first part of the statistical analysis included the distribution on age groups and the gender distribution in the study group and the control group. We also assessed the distribution of the main risk factors in the two groups.

Regarding the distribution on age groups the descriptive statistics revealed a mean age of 61.85 y.o in the study group and 71.28 y.o in the control group (Table 1).

TABLE 1. The mean age distribution in study group and control group

\begin{tabular}{|l|c|c|}
\hline Descriptive statistics \\
\hline & \multicolumn{2}{|c|}{ Age } \\
\hline Valid & Study & Control \\
\hline Missing & 39 & 43 \\
\hline Mean & 0 & 0 \\
\hline Median & 61.85 & 71.28 \\
\hline Mode & 64.00 & 74.00 \\
\hline Std. deviation & 68.00 & 77.00 \\
\hline IQR & 13.09 & 10.29 \\
\hline Minimum & 16.50 & 13.50 \\
\hline Maximum & 24.00 & 43.00 \\
\hline
\end{tabular}

This difference can be explained by the fact that the incidence of the contraindications for thrombolytic therapy increase with aging, which led to the inclusion of older patients in the control group.

In order to assess the statistical significance of the difference between the mean age in the two groups we evaluated the normal distribution of the datas with Shapiro-Wilk test and the test of equality of variances Levene (Table $2 \mathrm{~A}, \mathrm{~B}$ ).

TABLE 2 A,B. Shapiro-Wilk and Levene test for normality of age distribution in the two groups

A. Test of Normality (Shapiro-Wilk)

\begin{tabular}{|l|c|c|c|}
\hline & & W & p \\
\hline Age & Study & 0.93 & 0.02 \\
\hline & Control & 0.96 & 0.11 \\
\hline
\end{tabular}

B. Test of Equality of Variances (Levene's)

\begin{tabular}{|l|c|c|c|}
\hline & $\mathbf{F}$ & $\mathbf{d f}$ & $\mathbf{p}$ \\
\hline Age & 2.01 & 1 & 0.16 \\
\hline
\end{tabular}

Taking into account the unequal data distribution according to Levene test, we used Welch T-Test to assess the statistical significance of the difference between the two groups regarding mean age. The result proved to be statistical significant ( $p$ value $<0.001$ ), so the patients in the study group had a statistically significant lower mean age compared to control group (Table 3 ). 
TABLE 3. Welch test regarding the statistical significance of the difference between the mean age in the study group vs. control group

\begin{tabular}{|c|c|c|c|}
\hline \multicolumn{4}{|c|}{ Independent Samples T-Test } \\
\hline & $\mathbf{t}$ & $\mathbf{d f}$ & $\mathbf{p}$ \\
\hline Age & -3.60 & 72.05 & $<.001$ \\
\hline
\end{tabular}

The statistical analysis of gender distribution in the two groups revealed a frequency of women of $53.85 \%$ in study group vs. $58.14 \%$ in control group (Table 4).

TABLE 4. Contingency table regarding the gender distribution

\begin{tabular}{|l|c|c|c|}
\hline & \multicolumn{2}{|c|}{ Gender } & \\
\hline Group & F & M & Total \\
\hline Study & 21 & 18 & 39 \\
\hline Control & 25 & 18 & 43 \\
\hline Total & 46 & 36 & 82 \\
\hline
\end{tabular}

In order to assess the statistical significance of the difference between the two groups concerning gender distribution we used Chi-squared test. This test shows no statistical significance on the difference between the two groups on gender distribution (Table 5).

TABLE 5. Chi-squared test regarding the gender distribution in the two groups

\begin{tabular}{|l|c|c|c|}
\hline \multicolumn{4}{|c|}{ Chi-squared tests } \\
\hline & Value & df & $\mathbf{p}$ \\
\hline $\mathrm{X}^{2}$ & 0.15 & 1 & 0.70 \\
\hline $\mathrm{N}$ & 82 & & \\
\hline
\end{tabular}

The second element of the statistical analysis was represented by the assessment of the PESI score, both by its absolute value and by the distribution of the score elements in the two groups. The medium PESI score in the study group was lower than the control group (94.8 vs. 108, p value 0.02 ). This result is justified by the difference regarding mean age between the two groups and by the distribution of the PESI score elements. Therefore, the altered mental status was present in $12.82 \%$ of the patients in study group vs. $32.55 \%$ in control group (Table 6).

TABLE 6. Contingency table on altered mental status distribution in the two groups

\begin{tabular}{|l|c|c|c|}
\hline Group & Yes & No & Total \\
\hline Study & 5 & 34 & 39 \\
\hline Control & 14 & 29 & 43 \\
\hline Total & 19 & 63 & 82 \\
\hline
\end{tabular}

The Chi-squared test showed that this difference was statistical significant ( $\mathrm{p}$ value 0.03 ) (Table 7).
TABLE 7. The Chi-squared test on the statistical significance regarding the difference of altered mental status distribution in the two groups

\begin{tabular}{|l|c|c|c|}
\hline \multicolumn{4}{|c|}{ Chi-squared tests } \\
\hline & Value & df & $\mathbf{p}$ \\
\hline $\mathrm{X}^{2}$ & 4.48 & 1 & 0.03 \\
\hline $\mathrm{N}$ & 82 & & \\
\hline
\end{tabular}

This result is explained by the fact that altered mental status is associated with increasing age, meanwhile the mean age in control group being higher than study group.

The analysis of another PESI element, the systolic blood pressure on admission lower than $100 \mathrm{mmHg}$, showed that this criterion was met in $20.58 \%$ of patients in study group vs. $25.51 \%$ of patients in control group. The Chi-squared test showed no correlation with any group of this difference (Table 8 A, B).

TABLE 8 A, B. Contingency table and Chi-squared test on the distribution of SBP $<100 \mathrm{mmHg}$ in the two groups

A. Contingency tables

\begin{tabular}{|l|c|c|c|}
\hline & \multicolumn{2}{|c|}{ SBP $<\mathbf{1 0 0}$} & \\
\hline Group & Yes & No & Total \\
\hline Study & 8 & 31 & 39 \\
\hline Control & 11 & 32 & 43 \\
\hline Total & 19 & 63 & 82 \\
\hline
\end{tabular}

B. Chi-squared tests

\begin{tabular}{|l|c|c|c|}
\hline & Value & df & $\mathbf{p}$ \\
\hline $\mathrm{X}^{2}$ & 0.30 & 1 & 0.59 \\
\hline $\mathrm{N}$ & 82 & & \\
\hline
\end{tabular}

Following the same pattern of the statistical analysis we assessed the distribution of heart rate $(\mathrm{HR})>110 \mathrm{bpm}$ on admission in the two groups, this being another important element of the PESI score. There was no statistical significant difference between the two groups, as this factor was met in $41.03 \%$ of the patients from study group vs. $39.53 \%$ in control group. The Chi-squared test proved no statistical significance of this difference (Table 9 A,B).

TABLE 9 A, B. Contingency table and Chi-squared test on the distribution of HR $>110 \mathrm{bpm}$ on admission in the two groups

A. Contingency tables

\begin{tabular}{|l|c|c|c|}
\hline & \multicolumn{2}{|c|}{ Heart rate $\mathbf{1 1 0}$ bpm on admission } & \\
\hline Group & Yes & No & Total \\
\hline Study & 16 & 23 & 39 \\
\hline Control & 17 & 26 & 43 \\
\hline Total & 33 & 49 & 82 \\
\hline
\end{tabular}


B. Chi-squared tests

\begin{tabular}{|l|c|c|c|}
\hline & Value & df & df \\
\hline $\mathrm{X}^{2}$ & 0.02 & 1 & 0.89 \\
\hline $\mathrm{N}$ & 82 & & \\
\hline
\end{tabular}

The analysis of the incidence of chronic cardiac and pulmonary diseases (chronic obstructive pulmonary disease (COPD) and chronic congestive heart failure (CHF)) in the groups revealed a higher percent of these pre-existing medical conditions in the control group correlated with higher mean age. This difference did not meet the statistical significance, $(51.28 \%$ in study group vs. $60.47 \%$ in control group, $\mathrm{p}$ value 0.4 ) (Table $10 \mathrm{~A}, \mathrm{~B}$ ).

TABLE 10 A, B. Contingency table and Chi-squared test on the distribution of COPD and CHF in the two groups

\section{A. Contingency tables}

\begin{tabular}{|l|c|c|c|}
\hline & \multicolumn{2}{|c|}{ COPD, CHF } & \\
\hline Group & Yes & No & Total \\
\hline Study & 20 & 19 & 39 \\
\hline Control & 26 & 17 & 43 \\
\hline Total & 46 & 36 & 82 \\
\hline
\end{tabular}

\section{B. Chi-squared tests}

\begin{tabular}{|l|c|c|c|}
\hline & Value & df & df \\
\hline $\mathrm{X}^{2}$ & 0.70 & 1 & 0.40 \\
\hline $\mathrm{N}$ & 82 & & \\
\hline
\end{tabular}

To conclude, the difference between the medium PESI score in the two groups is the result of the mean age difference and of the higher incidence of altered mental status in the control group. However, both groups are included in the same PESI class, PESI III.

The third element of the statistical analysis was represented by the comparative assessment of early mortality and haemodynamical instability rates (in the first 7 days of admission) between the two groups. The clinical prognostic markers are the main determinants of the patient outcome, their assessment revealing the impact of the two therapies on the clinical evolution. Nevertheless, we also researched the major bleeding rates in the two groups, as defined by International Society of Hemostasis and Thrombosis (ISTH). The major bleedings are defined by fatal bleedings, bleedings affecting internal organs (brain, liver, intraspinal, retroperitoneal etc.), or associated with a decrease in hemoglobin level higher than $2 \mathrm{~g} / \mathrm{dl}$. This statistical element, related to the safety end-point of the therapy, represents an essential point in the proper selection of the patients to prevent increasing mortality and morbidity.

Regarding the early mortality rate - in the first 7 days from admission -, a statistical significant difference was found between the study group and the control group using Chi-squared test $(5.13 \%$ vs. $20.93 \%$, p value 0.04 ) (Table 11, Table $12 \mathrm{~A}, \mathrm{~B}$ ).

TABLE 11. Frequency table regarding mortality in the two groups

\begin{tabular}{|c|c|c|c|c|c|}
\hline \multicolumn{6}{|l|}{ Frequencies for mortality } \\
\hline Group & Mortality & Frequency & Percent & $\begin{array}{c}\text { Valid } \\
\text { percent }\end{array}$ & $\begin{array}{c}\text { Cumulative } \\
\text { percent }\end{array}$ \\
\hline Study & No & 37 & 94.87 & 94.87 & 94.87 \\
\hline & Yes & 2 & 5.13 & 5.13 & 100.00 \\
\hline & Missing & 0 & 0.00 & & \\
\hline & Total & 39 & 100.00 & & \\
\hline Control & No & 34 & 79.07 & 79.07 & 79.07 \\
\hline & Yes & 9 & 20.93 & 20.93 & 100.00 \\
\hline & Missing & 0 & 0.00 & & \\
\hline & Total & 43 & 100.00 & & \\
\hline
\end{tabular}

TABLE 12 A, B. Contingency table and Chi-squared test regarding comparative mortality rate

A. Contingency tables

\begin{tabular}{|l|c|c|c|}
\hline & \multicolumn{2}{|c|}{ mortality } & \\
\hline Group & $\mathbf{0}$ & $\mathbf{1}$ & Total \\
\hline Study & 37 & 2 & 39 \\
\hline Control & 34 & 9 & 43 \\
\hline Total & 71 & 11 & 82 \\
\hline
\end{tabular}

\section{B. Chi-squared tests}

\begin{tabular}{|l|c|c|c|}
\hline & Value & df & df \\
\hline $\mathrm{X}^{2}$ & 4.40 & 1 & 0.04 \\
\hline $\mathrm{N}$ & 82 & & \\
\hline
\end{tabular}

The statistical significant difference is sustained by pathophysiological elements, as the rate of pulmonary artery reperfusion is higher in thrombolysed patients compared to patients with classical treatment, in the data from large studies.

Assessing the early hemodynamic instability rate in the two groups, we found a lower incidence in the control group compared to control group $(10.26 \%$ vs. $30.23 \%)$. The difference was proved to be statistical significant using Chi-squared test ( $p$ value 0.03 ) (Table $13 \mathrm{~A}, \mathrm{~B}$ ).

TABLE 13 A, B. Contingency table and Chi-squared test on hemodynamic instability rate in the two groups

A. Contingency tables

\begin{tabular}{|l|c|c|c|}
\hline & \multicolumn{2}{|c|}{ Haemodynamical instability } & \\
\hline Group & $\mathbf{0}$ & $\mathbf{1}$ & Total \\
\hline Study & 35 & 4 & 39 \\
\hline Control & 30 & 13 & 43 \\
\hline Total & 65 & 17 & 82 \\
\hline
\end{tabular}

\section{B. Chi-squared tests}

\begin{tabular}{|l|c|c|c|}
\hline & Value & df & df \\
\hline $\mathrm{X}^{2}$ & 4.97 & 1 & 0.03 \\
\hline $\mathrm{N}$ & 82 & & \\
\hline
\end{tabular}


Finally, the analysis of the study safety endpoint regarding the major bleeding rate showed no statistical significant difference between the two groups. In the study group the major bleeding rate was $7.69 \%$ vs. $6.98 \%$ in the control group (Table $14 \mathrm{~A}$, B). This element is explained by the proper selection of the patients by including the high bleeding risk subjects in the control group.

TABLE 14 A, B. Contingency table and Chi-square test regarding the major bleedings in the two groups

A. Contingency tables

\begin{tabular}{|l|c|c|c|}
\hline & \multicolumn{2}{|c|}{ Major bleeding } & \\
\hline Group & Yes & No & Total \\
\hline Study & 3 & 36 & 39 \\
\hline Control & 3 & 40 & 43 \\
\hline Total & 6 & 76 & 82 \\
\hline
\end{tabular}

\section{B. Chi-squared tests}

\begin{tabular}{|l|c|c|c|}
\hline & Value & df & df \\
\hline $\mathrm{X}^{2}$ & 0.02 & 1 & 0.90 \\
\hline $\mathrm{N}$ & 82 & & \\
\hline
\end{tabular}

To conclude, there was noticed a statistical significant difference between the two groups referring to clinical prognostic markers - early mortality and hemodynamic instability -, which were lower in the thrombolysed group. However, the major bleedings rate was similar in the two groups, without a higher bleeding rate in the study group, because of the inclusion in the control group of patients with high bleeding risk.

\section{DISCUSSIONS}

In the present study we included 82 consecutive patients with intermediary-high risk acute PE, which were included in two groups, study group receiving thrombolytic therapy t-PA $100 \mathrm{mg}$ in $2 \mathrm{~h}$ and anticoagulation with UFH - and control group receiving UFH alone guided by aPTT. Patients with previous PE were excluded, also patients older than 85 years old were excluded because of the potential bleeding complications caused by anticoagulant therapy. The control group included patients with high bleeding risk - with contraindications for thrombolytic therapy, obese and subponderal patients, patients with severe renal impairment. The reason for this criterion was the data from previous studies which proved the high bleeding risk for these patients.

There was no difference between the two groups regarding the sex rate distribution (Table 4, Table $5)$, but the mean age in the control group was statistical significant higher compared to study group (Table 2, Table 3). The reason for this finding consists in the inclusion criteria, as most contraindications for thrombolytic therapy are related to older age.

The clinical prognostic markers analysis revealed a benefic effect of the thrombolytic therapy, with a lower early mortality and hemodynamic instability rate in the study group. The present data from previous studies regarding the impact on mortality and hemodynamic instability rate reveal discordant results. Chaterjee et al. evaluated in a metaanalysis the results of sixteen studies comparing the effect of thrombolytics compared to anticoagulation (10). Use of thrombolytics was associated with lower all-cause mortality (OR, $0.53 ; 95 \%$ CI, $0.32-0.88 ; 2.17 \%$ [23/1061] vs. $3.89 \%$ [41/1054] with anticoagulant and greater risks of major bleeding (OR, 2.73; 95\% CI, 1.91-3.91; 9.24\% [98/1061] vs. $3.42 \%$ [36/1054]. In this meta-analysis were included both patients with high risk and patients with intermediate-high risk. Compared to this metaanalysis the thrombolysed selected patients in our study had lower bleeding risk, this leading to the absence of a significant statistical difference regarding major bleeding.

Another meta-analysis was assessed by Wan et al. (12) and revealed no benefit in terms of mortality rate and bleeding risk. Eleven trials, involving 748 patients, were included in this meta-analysis. Compared with heparin, thrombolytic therapy was associated with a nonsignificant reduction in recurrent pulmonary embolism or death $(6.7 \%$ versus $9.6 \%$; OR $0.67,95 \%$ CI 0.40 to 1.12 , $\mathrm{P}$ for heterogeneity $=0.48)$, a nonsignificant increase in major bleeding $(9.1 \%$ versus $6.1 \%$; OR $1.42,95 \%$ CI 0.81 to 2.46), and a significant increase in nonmajor bleeding $(22.7 \%$ versus $10.0 \%$; OR $2.63,95 \%$ CI 1.53 to 4.54 ; number needed to harm $=8$ ). The main difference between the patients included in this study and the present study is that the patients were not selected to be at least at intermediate risk (patients with low risk were included). In our study only patients with intermediate-high risk were included.

Similarly, Dong et al. concluded that outcomes in terms of death rate, recurrent PE and haemorrhagic events were similar in patients who received thrombolytic therapy compared with placebo or heparin (13). The development of alternative therapeutic modalities for patients presenting with large-volume PE, with or without haemodynamic compromise, has been the subject of great interest. Whereas Jimenez et al. (14) concluded that recana- 
lisation procedures (full-dose, low-dose and catheter-assisted thrombolysis) did not offer a clear advantage in the treatment of PE compared with standard anticoagulation alone. In both these studies were also included patients with intermediate-low risk.

Another study evaluated the efficacy of half dose thrombolytic vs. anticoagulation alone in patients with moderate risk PE (15). During a 22-month period, 121 patients with moderate PE were randomized to receive a "safe dose" of tissue plasminogen activator plus anticoagulation (thrombolysis group [TG], $\mathrm{n}=61$ patients) or anticoagulation alone (control group [CG], $n=60$ ). The primary end points consisted of pulmonary hypertension and the composite end point of pulmonary hypertension and recurrent PE at 28 months. Pulmonary hypertension and the composite end point developed in 9 of 58 patients $(16 \%)$ in the TG and 32 of 56 patients $(57 \%)$ in the $\mathrm{CG}(\mathrm{p}<0.001)$ and 9 of 58 patients $(16 \%)$ in the TG and 35 of 56 patients $(63 \%)$ in the $\mathrm{CG}(\mathrm{p}<0.001)$, respectively. The secondary end points were total mortality, the duration of hospital stay, bleeding at the index hospitalization, recurrent PE, and the combination of mortality and recurrent PE. The duration of hospitalization was $2.2 \pm 0.5$ days in the TG and $4.9 \pm 0.8$ days in the CG $(p<0.001)$. The combination of death plus recurrent $\mathrm{PE}$ was $1(1.6 \%)$ in TG and $6(10 \%)$ in the $\mathrm{CG}(\mathrm{p}=0.0489)$. No bleeding occurred in any group, and despite a positive trend in favor of a "safe dose" thrombolysis, no significant difference was noted in the rate of individual outcomes of death and recurrent PE when assessed independently.

Meanwhile, the results from PEITHO multicentric study show a decrease in hemodynamic instability rate in thrombolysed patients (t-PA) compared to anticoagulation alone (9), but no influence regarding early mortality rate. The bleeding complications were higher in the study group, especially in patients over 80 y.o. (9). This study did not take into account the high bleeding risk associated with age over 80 y.o. and obese patients.

Another multi-centric study compared the halfdose t-PA with full dose t-PA. Progressive improvements in RVDs, lung perfusion defects, and pulmonary artery obstructions were found to be similarly significant in both treatment groups (16). This is true for patients with either hemodynamic instability or massive pulmonary artery obstruction. Compared with the $100 \mathrm{mg} / 2 \mathrm{~h}$ regimen, the $50 \mathrm{mg} / 2 \mathrm{~h}$ rt-PA regimen exhibits similar efficacy and perhaps better safety in patients with acute PTE. These findings support the notion that optimizing rt-PA dosing is worthwhile when treating patients with PTE. This study included patients with intermediate risk but also patients with severe obstruction of pulmonary arteries (without signs of clinical or imagistic severity). In our study we only included patients who met the criteria for intermediate-high risk PE, not taking into account the severity of pulmonary artery obstruction.

Goldhaber et al. (17) compared in two studies the effect of low dose bolus t-PA compared to 100 $\mathrm{mg}$ t-PA in $2 \mathrm{~h}$ (classic dose) in hemodynamic stable patients with PE. There were no differences between the reduced dose bolus and full dose $2 \mathrm{~h}$ rt-PA groups with respect to bleeding complications. Meanwhile, there was no difference regarding mortality rate between the two groups. Efficacy was similar in the two treatment groups. Compared to our study this study included hemodynamic stable patients, and did not compare the thrombolytic effects with the anticoagulant alone.

Meanwhile, a positive effect of thrombolytic therapy can be revealed in patients with right heart thrombi and intermediate PE (18), but this finding has to be supported by further data.

To conclude, the results of the present study show a positive effect of thrombolytic therapy compared to anticoagulation alone in patients with intermediate-high risk PE, while the present data in literature are discordant. This finding can be explained by a proper patients selection, by including patients with high bleeding risk in the non-thrombolysed group. Meanwhile, in most studies the included patients were not only in the intermediatehigh risk, as these studies included also patients with intermediate-low risk or high risk. The results for mortality rates can be justified by excluding patients older than 85 y.o. and patients with severe comorbidities which may influence independently the PE mortality.

The major bleeding rate (defined by the ISTH criteria) was not statistically significant different between the two groups, the safety endpoint of the study, as the higher bleeding risk patients were included in the control group. Regarding the patient selection for control group, we selected patients with higher bleeding risk in this group. Extreme BMI was associated with high bleeding risk in previous analysis. Mikola et al. proved in a meta-analysis including five studies that patients with a major bleeding complication were on average older than patients with no hemorrhagic complication (mean age $62.9+/-1.9$ years vs. $56.2+/-1.1$ years; $\mathrm{p}=0.005)$. By using age as a continuous variable, the study found a $4 \%$ (RR $1.04 ; 95 \%$ CI 1.02 to 
1.06) increase in risk of bleeding for each incremental year of age. In addition, patients with higher body mass index had an increased risk of bleeding (19).

In another study assessing the effect of thrombolytic therapy in patients with stroke, BMI was higher in patients with hemorrhagic transformation than in patients without hemorrhagic transformation. In addition, the odds ratio was 5.16 (95\% confidence interval, 2.21-12.04) when the body mass index was $\geq 25 \mathrm{~kg} / \mathrm{m}^{2}$ compared to a body mass index $<25 \mathrm{~kg} / \mathrm{m}^{2}(20)$.

The data from previous studies show a higher bleeding risk associated with thrombolytic therapy $(9,12)$, but these studies included also patients with extreme BMI and severe renal impairment, which can increase the bleeding risk as showed. The results from these studies is concordant with the result from another meta-analysis of 16 studies conducted during the last 45 years on thrombolysis for PE. Of 2115 patients, most (71\%) were classified as having intermediate-risk PE; 9.9\% had lowrisk PE; and $1.5 \%$ were hemodynamic unstable, high-risk PE. Risk could not be classified in the remaining patients. At mean follow-up of 82 days, all-cause mortality was significantly lower overall in patients who received thrombolytic therapy versus anticoagulant therapy ( $2.2 \%$ vs. $3.9 \%)$, but major bleeding was significantly higher $(9.2 \%$ vs. $3.4 \%)$, as was intracranial hemorrhage $(1.5 \%$ vs. $0.2 \%)$; results were similar when only patients with intermediate-risk PE were considered. Among patients older than 65, major bleeding was significantly more common with thrombolysis than with anticoagulant therapy (13\% vs. $4 \%$ ). Outcomes were no different in analyses that excluded the single trial that involved catheter-directed thrombolysis (20).

Another element that must be discussed is the apparently low number of patients, this element being justified by the inclusion criteria, excluding the medical conditions that may influence the patient prognosis and the elements that may worsen the pulmonary hypertension.

The patients over 85 y.o were excluded because of the high bleeding risk, as PEITHO study showed (9). Taking into account the high bleeding risk associated with thrombolytic therapy in patients with severe renal dysfunction, $\mathrm{BMI}<18.5 \mathrm{~kg} / \mathrm{m}^{2}$ or BMI $>30 \mathrm{~kg} / \mathrm{m}^{2}$ were excluded, also. Patients with contraindications for thrombolytic therapy were included in control group. As these elements are more frequent in older patients, the significant mean age difference is explained (61.85 y.o. study group vs. 71.28 y.o. control group). There was no significant difference between the two groups for gender distribution.

As weak points of the study we present the exclusive use of transthoracic echocardiography to assess the RV dysfunction and the RV pressure overload, this being explained by the method availability. The 3D echocardiography could not be used because of the low availability, especially in acute patients, as the pulmonary angio CT could not bring all the data for RV function assessment, the low benefit/cost ratio of angio $\mathrm{CT}$ being another reason for using transthoracic echocardiography.

Another weak point of the study is the follow up of the patients only in the acute period -7 days. The element that justifies this choice is the heterogeneity of the chronic treatment (the choice between vitamin $\mathrm{K}$ antagonists (VKA) and different novel anticoagulants (NOACs)), and the possible alteration of the impact that thrombolysis has on the prognosis of the patients by the type of oral anticoagulant followed. Also, the compliance to the anticoagulant treatment may influence the prognostic markers and may affect the rate of major bleedings.

Finally, we consider that the data base has to be improved by a higher number of patients, in order to explore the impact of the two therapies on the intermediate-high risk PE patient's outcome.

\section{CONCLUSIONS}

The present study reveals a statistically significant improvement of early mortality rate and hemodynamic instability rate in thrombolysed patients with intermediary-high risk PE, compared to patients treated with anticoagulants alone. This result is justified by patient selection, including patients with contraindications for thrombolysis and high bleeding risk in control group. As extreme BMI and severe renal dysfunction were associated with higher bleeding complications in thrombolysed patients in previous meta-analysis these patients were included in control group. By this study design there was no statistically significant difference between the two groups regarding the major bleeding rate. As this study is a single center one, the database is continuously developing, but higher number of patients is needed to analyse the effect of the two treatment protocols.

Conflict of interest: none declared Financial support: none declared 


\section{REFERENCES}

1. Cohen AT, Agnelli G, Anderson FA et al. VTE Impact Assessment Group in Europe (VITAE). Venous thromboembolism (VTE) in Europe. The number of VTE events and associated morbidity and mortality. Thromb Haemost. 2007;98:756-764.

2. Wendelboe AM, Raskob GE. Global burden of thrombosis: Epidemiologic aspects. Circ Res. 2016;118:1340-1347.

3. Jimenez D, de Miguel-Diez J, Guijarro R et al. Trends in the management and outcomes of acute pulmonary embolism: Analysis from the RIETE registry. J Am Coll Cardiol. 2016;67:162-170.

4. Agarwal S, Clark D III, Sud K, Jaber WA, et al. Gender disparities in outcomes and resource utilization for acute pulmonary embolism hospitalizations in the United States. Am J Cardiol. 2015; 116:1270-1276.

5. Roy PM, Meyer G, Vielle B et al. EMDEPU Study Group. Appropriateness of diagnostic management and outcomes of suspected pulmonary embolism. Ann Intern Med. 2006;144:157-164.

6. Jimenez D, Bikdeli B, Barrios D et al. RIETE Investigators. Management appropriateness and outcomes of patients with acute pulmonary embolism. Eur Respir J. 2018;51:1800445.

7. Wiener RS, Schwartz LM, Woloshin S. Time trends in pulmonary embolism in the United States: Evidence of overdiagnosis. Arch Intern Med. 2011;171:831-837.

8. Stavros V Konstantinides, Guy Meyer et al. 2019 ESC Guidelines for the diagnosis and management of acute pulmonary embolism developed in collaboration with the European Respiratory Society (ERS): The Task Force for the diagnosis and management of acute pulmonary embolism of the European Society of Cardiology (ESC). European Heart Journal 2020;41(4):543-603.

9. Meyer G, Vicaut E, Danays T et al. Fibrinolysis for patients with intermediate-risk pulmonary embolism. N Engl J Med. 2014; 370:1402-1411.

10. Chatterjee S, Chakraborty A, Weinberg I et al. Thrombolysis for pulmonary embolism and risk of all-cause mortality, major bleeding, and intracranial hemorrhage: A meta-analysis. JAMA. 2014;311:2414-2421.
11. Marti C, John G, Konstantinides S, Combescure C et al. Systemic thrombolytic therapy for acute pulmonary embolism: A systematic review and meta-analysis. Eur Heart J. 2015;36:605-614.

12. Wan S, Quinlan DJ, Agnelli G, et al. Thrombolysis compared with heparin for the initial treatment of pulmonary embolism: A metaanalysis of the randomized controlled trials. Circulation. 2004; 110(6):744-9.

13. Dong BR, Hao Q, Yue J, et al. Thrombolytic therapy for pulmonary embolism. Cochrane Database Syst Rev. 2009.

14. Jimenez D, Martin-Saborido C, Muriel A, et al. Efficacy and safety outcomes of recanalisation procedures in patients with acute symptomatic pulmonary embolism: systematic review and network meta-analysis. Thorax. 2018;73(5):464-71.

15. Sharifi M, Bay C, Skrocki L et al. Moderate pulmonary embolism treated with thrombolysis (from the "MOPETT" Trial). Am J Cardiol. 2013;111(2):273-7.

16. Wang $C$, Zhai Z, Yang $Y$ et al. Efficacy and safety of low dose recombinant tissue-type plasminogen activator for the treatment of acute pulmonary thromboembolism: A randomized, multicenter, controlled trial. Chest. 2010;137(2):254-62.

17. Goldhaber SZ, Feldstein ML, Sors H. Two trials of reduced bolus alteplase in the treatment of pulmonary embolism. An overview. Chest. 1994;106(3):725-6.

18. Burrage M, Gould P, McCann A: Half-Dose Thrombolysis in Submassive Pulmonary Embolism and Right-Heart Thrombus. Heart Lung Circ. 2016;25(Supplement 2).

19. Mikkola KM, Patel SR, Parker JA et al. Increasing age is a major risk factor for hemorrhagic complications after pulmonary embolism thrombolysis. Am Heart J. 1997;134(1):69-72.

20. Changqing Miao, Xiaoyan Yi, Chunying Mu, Yan Qu, Guogang Luo, Yurong Zhang. Body mass index is a risk factor for hemorrhagic transformation in older ischemic stroke patients after intravenous thrombolysis: A single center retrospective study. Research Square, November 2019. 